\title{
Characterization of DNase activity and gene in Streptococcus suis and evidence for a role as virulence factor
}

\author{
Bruno Haas', Laetitia Bonifait ${ }^{1}$, Katy Vaillancourt ${ }^{1}$, Steve J Charette ${ }^{2,3,4,5}$, Marcelo Gottschalk ${ }^{5,6}$ and Daniel Grenier ${ }^{1,5^{*}}$
}

\begin{abstract}
Background: The Gram-positive bacterium Streptococcus suis serotype 2 is an important swine pathogen and emerging zoonotic agent. Multilocus sequence typing allowed dividing S. suis serotype 2 into sequence types (STs). The three major STs of S. suis serotype 2 from North America are 1 (most virulent), 25 (intermediate virulence) and 28 (less virulent). Although the presence of DNase activity in S. suis has been previously reported, little data is available. The aim of this study was to investigate DNase activity in S. suis according to STs, to characterize the activity and gene, and to provide evidence for a potential role in virulence.

Results: We showed that ST1 and ST28 strains exhibited DNase activity that was absent in ST25 strains. The lack of activity in ST25 isolates was associated with a 14-bp deletion resulting in a shifted reading frame and a premature stop codon. The DNase of S. suis P1/7 (ST1) was cell-associated and active on linear DNA. A DNase-deficient mutant of $\mathrm{S}$. suis P1/7 was found to be less virulent in an amoeba model. Stimulation of macrophages with the DNase mutant showed a decreased secretion of pro-inflammatory cytokines and matrix metalloproteinase-9 compared to the parental strain.
\end{abstract}

Conclusions: This study further expands our knowledge of S. suis DNase and its potential role in virulence.

Keywords: Streptococcus suis, nuclease, DNase activity, Virulence factor, SsnA

\section{Background}

Streptococcus suis is an important swine pathogen worldwide that causes mainly meningitis, arthritis, endocarditis, and septicemia [1]. It can also affect humans in close contact with infected pigs or contaminated pork-derived products $[2,3]$. More particularly, two major S. suis outbreaks with symptoms of toxic shock-like syndrome occurred in 1998 and 2005 in China and caused over 50 deaths [4]. To date, thirty-five serotypes have been described and serotype 2 is the most commonly isolated from diseased pigs and humans [5]. In addition, S. suis is classified into numerous sequence types (STs) by multilocus sequence typing (MLST) [6]. A recent study showed

\footnotetext{
*Correspondence: daniel.grenier@greb.ulaval.ca

'Groupe de Recherche en Écologie Buccale (GREB), Faculté de Médecine Dentaire, Université Laval, 2420 Rue de la Terrasse, Quebec City, Quebec G1V OA6, Canada

${ }^{5}$ Centre de Recherche en Infectiologie Porcine et Avicole (CRIPA), Fonds de Recherche du Québec - Nature et Technologies (FRQNT), Saint-Hyacinthe,

Quebec, Canada

Full list of author information is available at the end of the article
}

that most isolates of S. suis serotype 2 from North America are part of three major STs: ST1, ST25 and ST28 showing high, intermediate and low virulence in a mouse model, respectively [7]. Despite several virulence factors already identified, the pathogenesis of $S$. suis infections is not fully understood, especially regarding the mechanisms that allow the bacterium to escape from the host immune system [8]. The best characterized factor providing resistance of $S$. suis to the host immune system is the sialic acid-rich capsule [8]. More specifically, the capsule confers resistance to phagocytosis by macrophages, and mutants deficient for capsule expression are less virulent in mouse and pig models of infection $[9,10]$. Moreover, unencapsulated mutants are also more sensitive to antibiotics, including penicillin $\mathrm{G}$ and ampicillin [11].

Evidence has been brought to support a role of bacterial deoxyribonucleases (DNases), which are enzymes that hydrolyze nucleic acids to yield oligonucleotides, as virulence factors. More specifically, DNases may be involved in bacterial growth [12] and biofilm maturation [13], as 
well as in the ability of bacteria to escape the immune system [14]. In 2004, a cell-associated DNase (112 kDa), encoded by the $\operatorname{ssn} A$ gene, has been identified in $S$. suis serotype 2 [15]. The recent pan-surfome analysis performed on S. suis and which concluded that the cellassociated DNase may represent one of the best vaccine candidates has revived the interest for this protein [16]. More specifically, it was found that the S. suis DNase is largely distributed among serotypes, and is highly immunogenic and accessible to antibodies [16]. In addition, de Buhr et al. [17] recently reported that S. suis DNase is involved in the degradation and escaping of neutrophil extracellular traps. The aim of the present study was to investigate the distribution of DNase activity among the three major STs (ST1, ST25, and ST28) of S. suis, to characterize the activity and gene, and to provide evidence for a potential role in the pathogenic process of $S$. suis infections.

\section{Methods}

\section{Bacterial strains and culture conditions}

Strains of S. suis used in this study and their origin are listed in Table 1. Bacteria were routinely grown in ToddHewitt Broth (THB) (BBL Microbiology Systems, Cokeysville, MD, USA) at $37^{\circ} \mathrm{C}$.

\section{Determination of DNase activity}

Strains of S. suis were screened for DNase activity using a plate assay. Briefly, $50 \mu \mathrm{l}$ of overnight broth cultures of S. suis were spotted on the surface of DNase Test Agar plates (BBL Microbiology Systems) which were incubated for $24 \mathrm{~h}$ at $37^{\circ} \mathrm{C}$. After adding $0.1 \mathrm{~N} \mathrm{HCl}$ for $5 \mathrm{~min}$, the appearance of a clear zone around bacterial growth indicated degradation of DNA. DNase activity of S. suis P1/7 was also determined and characterized by quantification of residual DNA after incubation of bacterial cells or filtered culture supernatants with DNA using the Quant-i $\mathrm{T}^{\mathrm{TM}}$ PicoGreen $^{\circledR}$ dsDNA Reagent (Invitrogen, Eugene, OR, USA) according to the manufacturer's protocol, as previously described [18], with some modifications. Briefly, linear double-stranded salmon sperm DNA (Sigma-Aldrich Canada Co., Oakville, ON, Canada) was prepared in DNA buffer (5.96 g/l HEPES [N-2-hydroxyethylpiperazine-N'-2ethanesulfonic acid], $590 \mathrm{mg} / \mathrm{l} \mathrm{CaCl}_{2} \cdot 2 \mathrm{H}_{2} \mathrm{O}$, and $380 \mathrm{mg} / \mathrm{l}$ $\mathrm{MgCl}_{2}, \mathrm{pH} 7.5$ prepared in RNase/DNase-free water) at $100 \mu \mathrm{g} / \mathrm{ml}$, and incubated with cells of S. suis $\left(\mathrm{OD}_{660}=1\right.$ in DNA buffer) or filtered culture supernatants. To determine heat stability of DNase activity, cells of S. suis were preheated for $30 \mathrm{~min}$ at 50,60 or $70^{\circ} \mathrm{C}$ prior to adding double-stranded salmon sperm DNA in the assay. A range of $\mathrm{pH}$ between 6 and 8 with increment of 0.5 was used to determine the optimal $\mathrm{pH}$ for DNase activity. Ion chelators (ethylenediaminetetraacetic acid [EDTA], ethylene glycol tetraacetic acid [EGTA] and 1,10-phenanthroline)
Table 1 Sequence type (ST), origin, and DNase activity of S. suis serotype 2 strains used in this study

\begin{tabular}{|c|c|c|c|}
\hline Strain & ST & Origin & DNase activity $^{1}$ \\
\hline $\mathrm{P} 1 / 7$ & 1 & Europe & + \\
\hline$M 2 D^{2}$ & $N A^{3}$ & NA & - \\
\hline MNCM01 & 1 & Thailand & + \\
\hline MNCM06 & 1 & Thailand & + \\
\hline MNCM16 & 1 & Thailand & + \\
\hline MGGUS2 & 1 & USA & + \\
\hline MGGUS3 & 1 & USA & + \\
\hline NIAH11433 & 1 & Japan & + \\
\hline DAT261 & 1 & Japan & + \\
\hline DAT264 & 1 & Japan & + \\
\hline DAT229 & 1 & Japan & + \\
\hline 1043248 & 25 & Canada & - \\
\hline 1043629 & 25 & Canada & - \\
\hline 1044423 & 25 & Canada & - \\
\hline 1053253 & 25 & Canada & - \\
\hline 1085543 & 25 & Canada & - \\
\hline MNCM04 & 25 & Thailand & - \\
\hline MNCM10 & 25 & Thailand & - \\
\hline MNCM24 & 25 & Thailand & - \\
\hline MNCM26 & 25 & Thailand & - \\
\hline MNCM51 & 25 & Thailand & - \\
\hline MGGUS4 & 25 & USA & - \\
\hline MGGUS5 & 25 & USA & - \\
\hline $89 / 1591$ & 25 & Canada & - \\
\hline 1054471 & 28 & Canada & + \\
\hline 1088563 & 28 & Canada & + \\
\hline 1097205 & 28 & Canada & + \\
\hline 1057906 & 28 & Canada & + \\
\hline MNCM43 & 28 & Thailand & + \\
\hline MGGUS9 & 28 & USA & + \\
\hline MGGUS10 & 28 & USA & + \\
\hline MGGUS11 & 28 & USA & + \\
\hline MGGUS12 & 28 & USA & + \\
\hline DAT292 & 28 & Japan & + \\
\hline DAT242 & 28 & Japan & + \\
\hline DAT245 & 28 & Japan & + \\
\hline DAT246 & 28 & Japan & + \\
\hline
\end{tabular}

${ }^{1}$ Determined with the DNase Test Agar medium. (+), presence of a clear zone around bacterial growth; (-), absence of a clear zone. ${ }^{2}$ DNase-deficient mutant from $S$. suis $\mathrm{P} 1 / 7$.

${ }^{3}$ Not applicable.

were tested for inhibition of S. suis DNase activity at 1 and $10 \mathrm{mM}$. Following incubation $\left(3 \mathrm{~h}\right.$ ) at $37^{\circ} \mathrm{C}$ (or 25,30 and $42^{\circ} \mathrm{C}$ for optimal temperature determination), samples were centrifuged $5 \mathrm{~min}$ at 11,000 $\mathrm{g}$ and residual DNA was 
quantified by adding the PicoGreen ${ }^{\circledR}$ reagent. After 5 min at room temperature, fluorescence was measured with a Synergy 2 BioTek microplate reader (BioTek Instruments, Inc, Winooski, VT, USA) using excitation wavelength of $485 \mathrm{~nm}$ and an emission wavelength of $528 \mathrm{~nm}$.

\section{Activity spectrum of S. suis DNase activity}

S. suis $\mathrm{P} 1 / 7$ cells from an overnight culture were harvested by centrifugation and suspended in $10 \mathrm{mM}$ Tris- $\mathrm{HCl}$ buffer ( $\mathrm{pH} 7.5$ ) containing $5 \mathrm{mM} \mathrm{CaCl}_{2}$ and $4 \mathrm{mM} \mathrm{MgCl}_{2}$ to an $\mathrm{OD}_{660}=1$. Cells were incubated with $40 \mathrm{ng} / \mu \mathrm{l}$ (final concentration) linear DNA (lambda DNA [Promega, Madison, WI, USA]) or $32.5 \mathrm{ng} / \mu \mathrm{l}$ (final concentration) circular plasmid DNA (pUC18) for $3 \mathrm{~h}$ at $37^{\circ} \mathrm{C}$ and degradation was monitored following migration in a $0.8 \%$ agarose gel $(45 \mathrm{~min}, 110 \mathrm{~V})$ and staining with EZ-Vision ${ }^{\mathrm{Tm}}$ Three (Amresco, Solon, OH, USA).

\section{Genotyping and sequence analysis}

Three strains of S. suis belonging to ST1 (P1/7, MNCM01, MGGUS2), ST25 (1043248, MNCM04, MGGUS4) and ST28 (1097205, MNCM43, MGGUS9) were grown for $24 \mathrm{~h}$ on THB agar plates. Colonies were suspended in sterile water, boiled for $10 \mathrm{~min}$ and centrifuged at 10,000 g for 3 min. PCR was then performed using the following primers in a reaction mixture containing $5 \mathrm{U} \mathrm{EconoTaq}^{\circ}$ (Lucigen, Middleton, WI) and $1.5 \mathrm{mM} \mathrm{MgCl}_{2}$ : $\mathrm{ssnA}$ 3088 F (5' GAC GTC CAT ATA TAA CAA AAA GGA G $3^{\prime}$ ) and ssnA 5392R (5' GTC GAT TCG GCC TAG GCT GAG ATT G 3') or ssnA 3884 F (5' ATT ACA GAA ACA AAC ATC GCT CAG T 3') and ssnA 6278R (5' ACA AGT GGA GGT GGA GCA GTA GAA A 3'). Thirty PCR cycles were performed starting with 1 min denaturation at $94^{\circ} \mathrm{C}, 1 \mathrm{~min}$ hybridization at $56^{\circ} \mathrm{C}$ and $3 \mathrm{~min}$ elongation at $72^{\circ} \mathrm{C}$. Products were sequenced and compared to the $s s n A$ sequence of $S$. suis P1/7 (ST1) using BioEdit and the NCBI database. BLASTp was used to compare the predicted amino acid sequence of the DNase with that of similar proteins identified in other streptococcal species.

\section{Isolation and characterization of a DNase deficient mutant}

A mutant library constructed in a previous study [19] by using the pTV408 temperature sensitive suicide vector to deliver the Tn917 transposon into S. suis $\mathrm{P} 1 / 7$ via electroporation was screened with the DNase plate assay to recover a deficient mutant. The number of transposon Tn917 insertion in the DNase deficient mutant (M2D) was determined by southern blot using a digoxygenin (DIG)-labeled probe specific to the erm gene of transposon Tn917 as previously described [20]. The exact site of insertion of transposon Tn917 in mutant M2D was determined using plasmid rescue. Briefly, genomic DNA was extracted and digested using HindIII, ligated and PCR was then performed on the ligature mix using the primers Erm-F (5' ACG AGT GAA AAA GTA CTC AAC C 3') and Tn917 (5' AGA GAG ATG TCA CCG TCA AGT 3'), $5 \mathrm{U}$ EconoTaq ${ }^{\oplus}$ (Lucigen) in $1.5 \mathrm{mM} \mathrm{MgCl}_{2}$. Thirty PCR cycles were performed starting with 1 min denaturation at $94^{\circ} \mathrm{C}$ followed by $1 \mathrm{~min}$ hybridization at $50^{\circ} \mathrm{C}$ and 5 min elongation at $72^{\circ} \mathrm{C}$. Amplicons were then sequenced (Genomic Analysis Platform of Université Laval, Québec, QC, Canada).

\section{Virulence assay in an amoeba model}

The DNase-deficient mutant (M2D) and its parental strain (P1/7) were tested using the amoeba host model Dictyostelium discoideum. In a previous study [21], we showed that $D$. discoideum cannot multiply and form plaques on lawns of virulent strains of S. suis, including P1/7, whereas plaque formation was observed on lawns of mutants (capsule, subtilisin-like protease) of S. suis known to be avirulent in an animal model. Briefly, amoebae were grown in HL5 liquid medium (14.3 g/l peptone, $7.15 \mathrm{~g} / \mathrm{l}$ yeast extract, $18 \mathrm{~g} / \mathrm{l}$ maltose, $3.6 \mathrm{mM} \mathrm{Na} 2 \mathrm{HPO}_{4}, 3.6 \mathrm{mM} \mathrm{KH_{2 }} \mathrm{PO}_{4}$ ) containing tetracycline $(15 \mu \mathrm{g} / \mathrm{ml})$. Amoebae were harvested by centrifugation ( $5 \mathrm{~min}$ at $1,500 \mathrm{~g}$ ), washed, and resuspended in tetracycline-free HL5 medium at a concentration of $300,150,75,38,18$, or 9 amoeba cells per $5 \mu \mathrm{l}$. Meanwhile, bacteria were harvested by scraping the surface of two Petri plates with confluent lawns of growth (overnight at $37^{\circ} \mathrm{C}$ ) and suspended in $2 \mathrm{ml}$ of HL5 medium without tetracycline. The bacterial suspensions $(100 \mu \mathrm{l}$, $\mathrm{OD}_{660} \approx 5$ ) were then applied to wells of 24-well plates containing HL5 agar medium ( $2 \mathrm{ml} /$ well). Once dried, bacterial lawns were spotted with $5 \mu \mathrm{l}$ of the $D$. discoideum suspensions. Plates were incubated at $23^{\circ} \mathrm{C}$ for 2 days and then examined visually for plaque formation. Three independent experiments were performed to ensure reproducibility.

\section{Cytokine and MMP-9 secretion by a macrophage model}

The human monoblastic leukemia cell line U937 (ATCC CRL-1593.2; American Type Culture Collection, Manassas, VA, USA) was cultivated at $37^{\circ} \mathrm{C}$ in a $5 \% \mathrm{CO}_{2}$ atmosphere in RPMI-1640 medium (HyClone Laboratories, Logan, UT, USA) supplemented with $10 \%$ heat-inactivated fetal bovine serum (FBS; RPMI-FBS) and $100 \mu \mathrm{g} / \mathrm{ml}$ penicillin-streptomycin. Differentiation into macrophage-like cells with phorbol 12-myristate 13-acetate was performed as previously described [22]. One million macrophage-like cells were seeded in 12-wells culture plates and incubated overnight at $37^{\circ} \mathrm{C}$ in $5 \% \mathrm{CO}_{2}$. Culture medium was then aspirated and replaced with fresh RPMI-1640 supplemented with $1 \%$ heat-inactivated FBS and $100 \mu \mathrm{g} / \mathrm{ml}$ penicillinstreptomycin containing S. suis (DNase-deficient mutant [M2D] or parental strain [P1/7]) at multiplicity of infection (MOI) of 100,50 or 10 . After $24 \mathrm{~h}$ of stimulation at $37^{\circ} \mathrm{C}$ 
in $5 \% \mathrm{CO}_{2}$, supernatants were collected. Quantification of interleukin-6 (IL-6), interleukin-8 (CXCL8), tumor necrosis factor- $\alpha$ (TNF- $\alpha$ ), and matrix metalloproteinase 9 (MMP-9) was performed by enzyme-linked immunosorbent assays (ELISA) (eBioscience Inc., San Diego, CA, USA) following the manufacturer's instructions. All treatments were performed in triplicate. Differences between means were analyzed for statistical significance using the Student's t-test and were considered significant at $p<0.05$.

\section{Results}

Distribution of DNase activity and comparative analysis of ssnA gene in S. suis according to STs

Thirty-six $S$. suis serotype 2 strains were tested for DNase activity using the plate assay. All strains grew similarly on the DNase Test Agar medium. As reported in Table 1, strains belonging to ST1 and ST28 all possessed DNase activity whereas ST25 strains did not show any capacity to degrade DNA. A comparative analysis of the $\operatorname{ssn} A$ gene of $S$. suis strains belonging to either ST1, ST25 or ST28 was performed. Sequence comparison of the $s s n A$ gene of S. suis P1/7 (ST1; SSU1760; UniProt accession no. C5VVJ6) with those amplified from $S$. suis serotype 2 belonging to each of the three STs showed that the nucleic acid sequence of $\operatorname{ssn} A$ gene of $S$. suis $\mathrm{P} 1 / 7$ is identical to that of corresponding genes found in $S$. suis isolates belonging to ST1 group. The $\operatorname{ssn} A$ gene from $\mathrm{P} 1 / 7$ showed a $\%$ identity of $98.1 \%$ and $98 \%$ with corresponding genes in strains of the ST25 and ST28 groups, respectively. However, when the predicted amino acid sequences were analyzed, the ST25 group (devoid of DNase activity) showed only $36.3 \%$ identity with the predicted protein of gene SSU1760 of S. suis P1/7 whereas ST28 and ST1 groups showed $98.4 \%$ and $100 \%$ identity, respectively. A detailed sequence analysis revealed that the low \% identity in the predicted protein of $S$. suis $\operatorname{ssn} A$ gene of the ST25 group is related to a truncated form of the protein due to the loss of $14 \mathrm{bp}$ region starting from position 1140 (Figure 1). A shifted reading frame is associated with this deletion, generating a stop codon starting at position 1170 .

\section{Genetic organization of ssnA gene (SSU1760)}

The $\operatorname{ssn} A$ gene is preceded by gsh $A$ (glutamate cysteine ligase/glutathione synthetase) and followed by hslO (Hsp33like chaperonin). This genetic organization is conserved in all published genomes of $S$. suis (data not shown). The open reading frame (ORF) of $S$. suis $\mathrm{P} 1 / 7$ ssn $A$ is $3120 \mathrm{bp}$ in length and codes for a 1039 amino acid protein. The resulting SsnA protein possesses the Gram positive cell wall anchoring motif (LPKTG) at the C-terminus end followed by a hydrophobic domain, while a signal peptide is found at the N-terminus end. It also has two oligonucleotide/oligosaccharide-binding (OB)-fold domains. The active site belongs to the endonuclease/exonuclease/phosphatase family domain according to Kegg database (http://www.genome. jp/dbget-bin/www_bget?ssi:SSU1760).

\section{Percentage identity of the DNase SsnA (SSU1760) with similar proteins found in pathogenic streptococci} Comparison of the predicted amino acid sequence of DNase SsnA (SSU1760) with similar proteins found in other pathogenic streptococcal species showed that the protein shares 48.7\% and 48.4\% identity with SpnA and spyM18_0808, respectively, which are both cell-wall anchored DNases of Streptococcus pyogenes. The predicted nucleic acid-binding domain (ITETNIAQLATQAQATLVSLKN) identified by Fontaine et al. [15] in the DNase contains several residues found in the S. pyogenes SpnA nucleic acid-binding domain (VKEAVISELETTTPSTLVKLSH).

\section{Characterization of S. suis P1/7 DNase activity}

The fluorogenic assay was used to determine the localization, stability and optimal conditions of DNase activity of S. suis P1/7 (Table 2). The activity was found to be cell-associated and not secreted in the culture supernatant. S. suis DNase activity was heat sensitive since less than $2 \%$ activity remained after treatment of bacteria at 
Table 2 Sublocalization, stability, optimal conditions and inhibition of DNase activity of S.suis P1/7

\begin{tabular}{ll}
\hline Treatment/condition & Activity (\%) \pm SD \\
\hline Sublocalization & \\
Pellets & $100 \pm 0.2$ \\
Supernatant & $7.2 \pm 1.0$ \\
Stability & \\
No treatment & $100 \pm 0.2$ \\
$50^{\circ} \mathrm{C} / 30$ min & $1.8 \pm 0.3$ \\
$60^{\circ} \mathrm{C} / 30 \mathrm{~min}$ & $1.9 \pm 1.0$ \\
$70^{\circ} \mathrm{C} / 30 \mathrm{~min}$ & $0 \pm 1.8$ \\
Reactional pH & \\
6.0 & $38.5 \pm 0.8$ \\
6.5 & $82.1 \pm 0.5$ \\
7.0 & $100 \pm 0.2$ \\
7.5 & $98.7 \pm 0.1$ \\
8.0 & $96.4 \pm 0.2$ \\
Incubation temperature & \\
$25^{\circ} \mathrm{C}$ & \\
$30^{\circ} \mathrm{C}$ & $10.0 \pm 0.2$ \\
$37^{\circ} \mathrm{C}$ & $49.3 \pm 0.3$ \\
$42^{\circ} \mathrm{C}$ & $100 \pm 0.1$ \\
Inhibition & $110.6 \pm 0.05$ \\
None & \\
EDTA $10 \mathrm{mM}$ & \\
EGTA $10 \mathrm{mM}$ & $100 \pm 13.0$ \\
EGTA $1 \mathrm{mM}$ & $0 \pm 7.1$ \\
\hline, $10-$ phenanthroline $10 \mathrm{mM}$ & $46.8 \pm 9.7$ \\
\end{tabular}

$50^{\circ} \mathrm{C}$ (30 min). The optimal $\mathrm{pH}$ for the activity ranged between $\mathrm{pH} 7$ and 7.5. DNase activity was slightly stronger at a temperature of $42^{\circ} \mathrm{C}$ than $37^{\circ} \mathrm{C}$. The activity was completely inhibited by EDTA (10 mM) and EGTA (10 and $1 \mathrm{mM}$ ) and partially inhibited by 1,10-phenanthroline. To determine the substrate specificity of $S$. suis DNase, linear DNA or circular plasmid DNA was incubated with bacterial cells prior to monitor degradation by electrophoresis. As shown in Figure 2, the band corresponding to linear DNA was degraded after incubation with S. suis P1/7 compared to the control without bacteria (compare lane 3 with lane 2). The migration profile of circular DNA alone (lane 5) showed 3 distinct bands, which correspond to nicked DNA (6 kb band), linear DNA (4 kb band) and supercoiled DNA (2.5 kb band) [23]. Incubation of circular DNA with the bacteria (lane 6) caused the appearance of a new band at $2.6 \mathrm{~kb}$ compared to the control sample. This band could correspond to supercoiled DNA bound

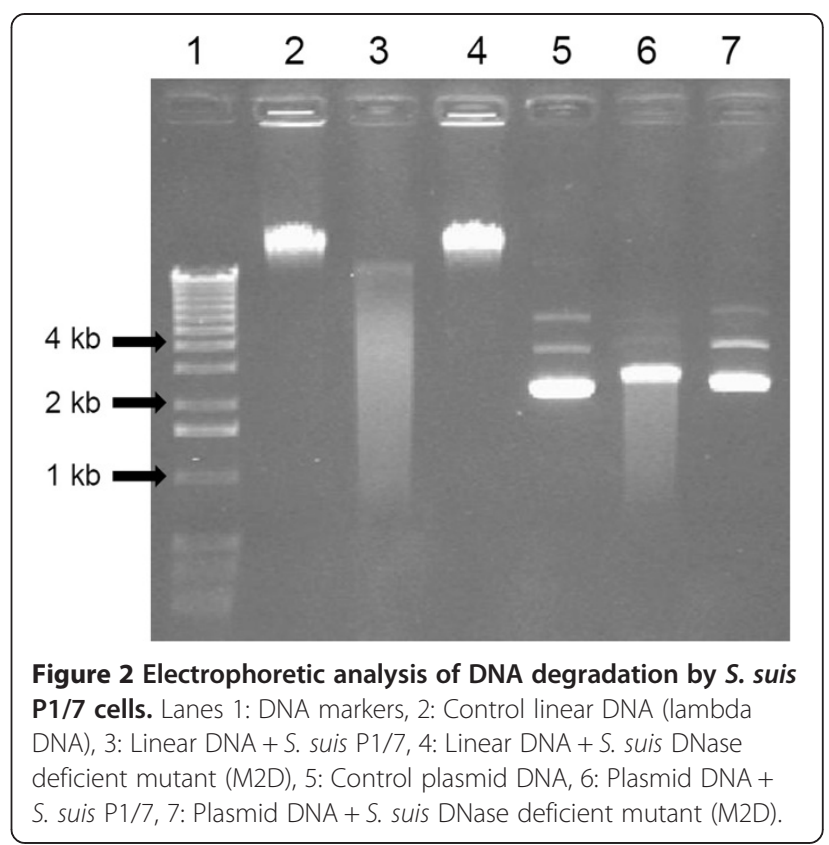

to the DNase [23]. A decreased intensity of the two bands ( $4 \mathrm{~kb}$ and $6 \mathrm{~kb}$ ) corresponding to the linear and nicked forms of the plasmid was observed.

\section{Isolation of a DNase-deficient mutant from a bank of mutants}

A bank of mutants $(1,150)$ of $S$. suis $\mathrm{P} 1 / 7$ prepared in a previous study [19] by insertion of transposon Tn917 was screened using the DNase Test Agar medium, and one mutant (M2D) completely devoid of DNase activity was identified. The loss of DNase activity was also confirmed after incubation of linear DNA with the mutant M2D (Figure 2, lane 4). Southern blot analysis of genomic DNA was performed in order to determine the number of insertion in mutant M2D. A single band was identified supporting the presence of one insertion of transposon Tn917 (data not shown). Using a plasmid rescue procedure, the insertion causing the loss of DNase activity was found to be in position 762 in $\operatorname{ssn} A$ gene.

\section{Virulence of the DNase-deficient mutant in an amoeba virulence model}

The virulence assay using the amoeba model $D$. discoideum was used to compare $S$. suis P1/7 with its DNase-deficient mutant M2D. As reported in Table 3, $\geq 300$ amoebae per well were needed to cause plaque formation on bacterial lawns of the parental strain. However, amoebae in the range of 9 to 18 cells per well were sufficient to induce plaque formation on lawns of the DNase-deficient mutant, thus indicating a much higher susceptibility to predation by D. discoideum. 
Table 3 Susceptibility of S. suis P1/7 and its DNase-deficient mutant (M2D) to predation of $D$. discoideum

\begin{tabular}{lcccc}
\hline Strain & $\begin{array}{c}\text { DNase } \\
\text { activity }\end{array}$ & Expt $\mathbf{1}$ & Expt $\mathbf{2}$ & Expt 3 \\
\hline $\mathrm{P} 1 / 7$ & + & $>300$ & $>300$ & 300 \\
$\mathrm{M} 2 \mathrm{D}$ & - & 18 & 9 & 9 \\
\hline
\end{tabular}

Inflammatory response induced by the DNase-deficient mutant in a macrophage model

The impact of the loss of DNase activity in the inflammatory response of $S$. suis cells in a macrophage model was investigated. Both $S$. suis $\mathrm{P} 1 / 7$ and its DNasedeficient mutant M2D induced IL-6, CXCL8, TNF- $\alpha$ and MMP-9 secretion by macrophages in a dose-dependent fashion (Figure 3). However, macrophages stimulated with cells of mutant M2D secreted significantly lower amounts of cytokines and MMP-9 in comparison with the parental strain. More specifically, at MOI of 100, the cells stimulated with mutant M2D produced $65.2 \%$, $31.6 \%, 44.7 \%$ and $19.8 \%$ less IL-6, CXCL8, TNF- $\alpha$ and MMP-9 respectively, than the parental strain P1/7.

\section{Discussion}

$S$. suis serotype 2 is responsible for important economical losses in the swine industry worldwide. In order to better understand the pathogenic process of S. suis infections, it is essential to identify all virulence factors produced by this pathogen and to evaluate their exact contribution in pathogenesis. In a previous study, Fontaine et al. [15] identified a cell wall-anchored DNase in S. suis. Given that DNases have been suggested as an important virulence factor in human pathogenic streptococci $[24,25]$ and that very few is known on the DNase of S. suis, this study is of relevance. We first investigated the presence of this activity in the three major North American STs, namely ST1, ST25 and ST28 that differ in their virulence in a mouse model [7]. This comparative analysis suggests that a direct link between virulence in mice and the presence of DNase activity cannot be established. While strains belonging to the most virulent ST (ST1) and to the less virulent ST (ST28) were all positive for DNase activity, ST25 strains having an intermediate virulence lacked the activity. The absence of DNase activity in ST25 strains is explained by a 14-bp deletion from position 1140 to 1153 in $s s n A$ gene of these strains causing a shifted reading frame that generates a stop codon starting at position 1170 .

Considering that the insertion of a single transposon in mutant M2D resulted in a complete loss of DNase activity, it can be suggested that the DNase encoded by the $\operatorname{ssn} A$ gene is the only active extracellular DNase expressed by S. suis in the tested conditions. Interestingly, twenty-five genes coding for putative nucleases, including fourteen DNases are present in S. suis P1/7 genome (data not shown). Ones should not exclude that additional DNases
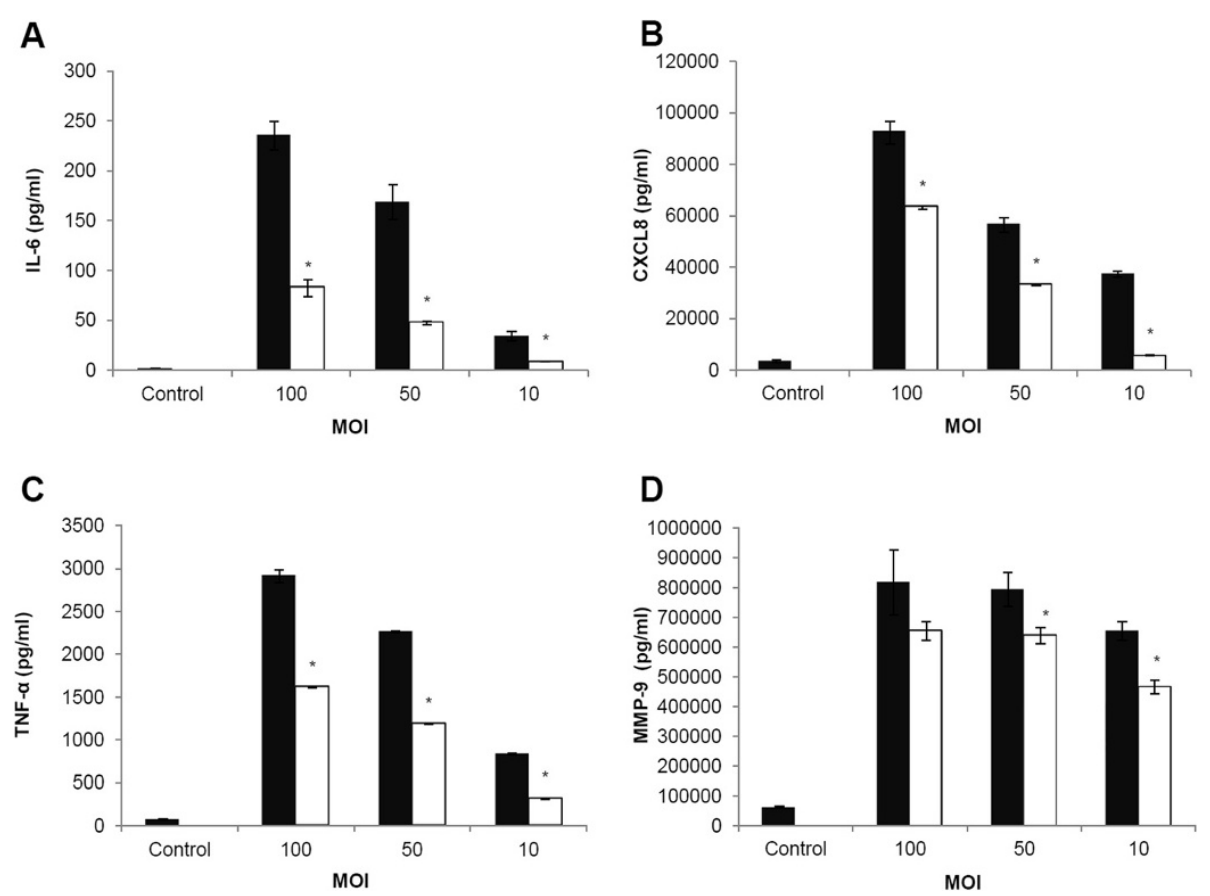

Figure 3 Quantification of pro-inflammatory cytokines and MMP-9 produced by macrophages stimulated with S. suis P1/7 and M2D. Secretion of IL-6 (panel A), CXCL8 (panel B), TNF-a (panel C) and MMP-9 (panel D) by macrophages stimulated with cells of S. suis P1/7 and its DNase-deficient mutant M2D at MOls of 10, 50, and 100. *: $p<0.05$. . S. suis P1/7, $\square$ S. suis M2D. 
strictly located in the cytoplasm and contributing to DNA catabolism and/or reparation can be expressed in addition to other extracellular DNase(s) only under specific conditions. No data are available on the localization of these putative nucleases.

The ability of S. suis DNase to degrade linear and not circular DNA, as stated previously [15], suggests that it has uniquely an exonuclease activity. The fact that circular DNA treated with $S$. suis P1/7 showed a decreased intensity of the bands corresponding to the nicked and linear forms of the plasmid could be the result of its partial degradation. The remaining band migrated at $2.6 \mathrm{~kb}$ rather than $2.5 \mathrm{~kb}$ in the control sample could correspond to the binding of the DNase to supercoiled DNA [23]. As opposed to the findings of Fontaine et al. [15], we found that $S$. suis P1/7 DNase was heat-sensitive. This discrepancy may be related to the fact that we used a more sensitive assay procedure to determine DNase activity. S. suis DNase was active at physiological $\mathrm{pH}$ and temperature, with an increased activity at $42^{\circ} \mathrm{C}$ suggesting that, in case of infection, fever might promote DNase activity. Inhibition of the activity by ion chelators such as EDTA, EGTA or 1,10-phenanthroline is in agreement with the fact that the DNase activity of $S$. suis depends on the presence of $\mathrm{Ca}^{2+}$ and $\mathrm{Mg}^{2+}$ [15].

D. discoideum amoeba has been shown to be a suitable alternative host model to analyze the virulence of various pathogenic bacteria mainly due to its phagocytic activity and its easiness to use [26,27], prior to use an animal model. In a previous study, we showed that $S$. suis mutants deficient for capsule expression or for the production of the subtilisin protease were sensitive to amoeba predation suggesting a decreased virulence as the one seen in an animal model [21]. In this study, the DNasedeficient mutant was found to be more susceptible to amoeba predation compared to its parental strain. This suggests that DNase activity likely contributes to the virulence of S. suis, probably through an action on the phagocytic cells. Therefore, this decreased virulence should be confirmed in an animal model such as mouse or piglet.

To determine a potential contribution of S. suis DNase in the host inflammatory response, macrophages were stimulated with the DNase-deficient mutant and its parental strain and cytokine and MMP-9 secretion was monitored. It was found that macrophages stimulated with $S$. suis P1/7 secreted higher amounts of IL-6, CXCL8, TNF- $\alpha$, and MMP-9 than those stimulated with M2D. To the best of our knowledge, this is the first report on the ability of a bacterial DNase to exert a pro-inflammatory effect on macrophages. This contribution of $S$. suis DNase in cytokine and MMP-9 secretion may be of utmost importance in the pathogenic process of meningitis. Indeed, Lopes-Cortes et al. [28] reported that TNF- $\alpha$ is present in the cerebrospinal fluid and that high levels of this cytokine correlate with neurological complications. Moreover, high concentrations of MMP-9 are observed in vivo in the cerebrospinal fluid during bacterial meningitis and in an experimental model of meningitis $[29,30]$. These results in addition to the one from the amoeba virulence assay are evidences that the $S$. suis DNase activity directly affects phagocytic cells confronted to the bacterium. DNases have often been described as virulence factors in streptococci [31] or staphylococci [14]. Indeed, it has been shown that DNase can help bacteria to escape from neutrophil extracellular traps (NETs) which are structures secreted by neutrophils to trap and kill bacteria [32]. These structures are mainly made of DNA, proteases, anti-microbial peptides and histones [33]. A recent study showed that $S$. suis DNase can degrade NETs to escape from the host innate immune system [17].

\section{Conclusions}

In this study, we further characterized at the genetic and functional levels the $S$. suis cell wall-anchored DNase. We showed that this activity was present in all virulent strains belonging to ST1 and that less virulent isolates (ST25) lacked the activity. The absence of DNase activity was due to a conserved 14-bp deletion in the $\operatorname{ssn} A$ gene causing a shifted reading frame and apparition of an early stop codon resulting in a truncated and inactive protein. We showed that the DNase is active at physiological temperature and $\mathrm{pH}$ on linear DNA, and also brought evidences that it represents the most active DNase of S. suis since its inactivation completely abolished $S$. suis DNase activity. Furthermore, the DNase may contribute to the virulence of $S$. suis by increasing the inflammatory response.

\section{Competing interests}

The authors declare that there is no competing interests regarding the publication of this article.

\section{Authors' contributions}

$\mathrm{BH}$ was the main experimentator for this study while LB carried out the screening of the S. suis P1/7 mutant library, isolated the DNase-deficient mutant and performed part of the virulence assays. KV was in charge of the molecular biology assays and analysis. SJC supervised the virulence assay. MG provided the S. suis strains. DG supervised and conceived this study. $\mathrm{BH}$ prepared the manuscript under the supervision of DG. All authors read and approved the manuscript.

\section{Acknowledgments}

We wish to thank J. Slater (The Royal Veterinary College, Hatfield, United Kingdom) for providing the S. suis mutant bank. This study was funded by Discovery grants to DG, MG, and SJC from the Natural Sciences and Engineering Research Council of Canada (NSERC). SJC is a research scholar of the Fonds de recherche du Québec - Santé (FRQS). BH is recipient of a scholarship from the Swine and Poultry Infectious Disease Research Center (CRIPA).

\section{Author details}

'Groupe de Recherche en Écologie Buccale (GREB), Faculté de Médecine Dentaire, Université Laval, 2420 Rue de la Terrasse, Quebec City, Quebec G1V OA6, Canada. ${ }^{2}$ Institut de Biologie Intégrative et des Systèmes (IBIS), Quebec City, Quebec, Canada. ${ }^{3}$ Centre de Recherche de I'Institut Universitaire de 
Cardiologie et de Pneumologie de Québec (CRIUCPQ), Hôpital Laval, Quebec City, Quebec, Canada. ${ }^{4}$ Département de Biochimie, de Microbiologie et de Bio-Informatique, Faculté des Sciences et de Génie, Université Laval, Quebec City, Quebec, Canada. ${ }^{5}$ Centre de Recherche en Infectiologie Porcine et Avicole (CRIPA), Fonds de Recherche du Québec - Nature et Technologies (FRQNT), Saint-Hyacinthe, Quebec, Canada. ${ }^{6}$ Groupe de Recherche sur les Maladies Infectieuses du Porc (GREMIP), Faculté de Médecine Vétérinaire, Université de Montréal, Saint-Hyacinthe, Quebec, Canada.

\section{Received: 7 April 2014 Accepted: 27 June 2014}

Published: 4 July 2014

\section{References}

1. Gottschalk M: Streptococcosis. In Diseases of swine. Edited by Karriger LRA, Schwartz K, Stevenson G, Zimmerman J. NJ, USA: Wiley Publishers; 2012:841-855.

2. Gottschalk M, Segura M, Xu J: Streptococcus suis infections in humans: the Chinese experience and the situation in North America. Anim Health Res Rev 2007, 8:29-45.

3. Wertheim HFL, Nghia HDT, Taylor W, Schultsz C: Streptococcus suis: an emerging human pathogen. Clin Infect Dis 2009, 48:617-625.

4. Yu H, Jing H, Chen Z, Zheng H, Zhu X, Wang H, Wang S, Liu L, Zu R, Luo L, Xiang N, Liu H, Liu X, Shu Y, Lee SS, Chuang SK, Wang Y, Xu J, Yang W: Human Streptococcus suis outbreak, Sichuan, China. Emerg Infect Dis 2006, 12:914-920

5. Staats JJ, Feder I, Okwumabua O, Chengappa MM: Streptococcus suis: past and present. Vet Res Commun 1997, 21:381-407.

6. King SJ, Leigh JA, Heath PJ, Luque I, Tarradas C, Dowson CG, Whatmore AM: Development of a multilocus sequence typing scheme for the pig pathogen Streptococcus suis: identification of virulent clones and potential capsular serotype exchange. J Clin Microbiol 2002, 40:3671-3680.

7. Fittipaldi N, Xu JG, Lacouture S, Tharavichitkul P, Osaki M, Sekizaki T, Takamatsu D, Gottschalk M: Lineage and virulence of Streptococcus suis serotype 2 isolates from North America. Emerg Infect Dis 2011, $17: 2239-2244$

8. Fittipaldi N, Segura M, Grenier D, Gottschalk M: Virulence factors involved in the pathogenesis of the infection caused by the swine pathogen and zoonotic agent Streptococcus suis. Future Microbiol 2012, 7:259-279.

9. Charland N, Harel J, Kobisch M, Lacasse S, Gottschalk M: Streptococcus suis serotype 2 mutants deficient in capsular expression. Microbiology 1998, 144(Pt 2):325-332.

10. Smith HE, Damman M, van der Velde J, Wagenaar F, Wisselink HJ, Stockhofe-Zurwieden N, Smits MA: Identification and characterization of the cps locus of Streptococcus suis serotype 2: the capsule protects against phagocytosis and is an important virulence factor. Infect Immun 1999, 67:1750-1756.

11. Tanabe SI, Bonifait L, Fittipaldi N, Grignon L, Gottschalk M, Grenier D: Pleiotropic effects of polysaccharide capsule loss on selected biological properties of Streptococcus suis. Can J Vet Res 2010, 74:65-70.

12. Mulcahy $\mathrm{H}$, Charron-Mazenod L, Lewenza S: Pseudomonas aeruginosa produces an extracellular deoxyribonuclease that is required for utilization of DNA as a nutrient source. Environ Microbiol 2010, 12:1621-1629.

13. Mann EE, Rice KC, Boles BR, Endres JL, Ranjit D, Chandramohan L, Tsang LH, Smeltzer MS, Horswill AR, Bayles KW: Modulation of eDNA release and degradation affects Staphylococcus aureus biofilm maturation. PLOS ONE 2009, 4:e5822.

14. Berends ETM, Horswill AR, Haste NM, Monestier M, Nizet V, von KockritzBlickwede M: Nuclease expression by Staphylococcus aureus facilitates escape from neutrophil extracellular traps. J Innate Immun 2010, 2:576-586.

15. Fontaine MC, Perez-Casal J, Willson PJ: Investigation of a novel DNase of Streptococcus suis serotype 2. Infect Immun 2004, 72:774-781.

16. Gomez-Gascon L, Luque I, Olaya-Abril A, Jimenez-Munguia I, OrbegozoMedina RA, Peralbo E, Tarradas C, Rodriguez-Ortega MJ: Exploring the pan-surfome of Streptococcus suis: looking for common protein antigens. J Proteomics 2012, 75:5654-5666.

17. de Buhr N, Neumann A, Jerjomiceva N, von Kockritz-Blickwede M, Baums CG. Streptococcus suis DNase SsnA contributes to degradation of neutrophil extracellular traps (NETs) and evasion of NET-mediated antimicrobial activity. Microbiology 2014, 160:385-395.
18. Choi SJ, Szoka FC: Fluorometric determination of deoxyribonuclease I activity with PicoGreen. Anal Biochem 2000, 281:95-97.

19. Slater JD, Allen AG, May JP, Bolitho S, Lindsay H, Maskell DJ: Mutagenesis of Streptococcus equi and Streptococcus suis by transposon Tn917. Vet Microbiol 2003, 93:197-206.

20. Bonifait L, Dominguez-Punaro MD, Vaillancourt K, Bart C, Slater J, Frenette M, Gottschalk M, Grenier D: The cell envelope subtilisin-like proteinase is a virulence determinant for Streptococcus suis. BMC Microbiol 2010, 10:42.

21. Bonifait L, Charette SJ, Filion G, Gottschalk M, Grenier D: Amoeba host model for evaluation of Streptococcus suis virulence. Appl Environ Microb 2011, 77:6271-6273.

22. Bonifait L, Grenier D: The SspA subtilisin-like protease of Streptococcus suis triggers a pro-inflammatory response in macrophages through a non-proteolytic mechanism. BMC Microbiol 2011, 11:47.

23. Gadi J, Ruthala K, Kong KA, Park HW, Kim MH: The third helix of the Hoxc8 homeodomain peptide enhances the efficiency of gene transfer in combination with lipofectamine. Mol Biotechnol 2009, 42:41-48.

24. Hasegawa T, Minami M, Okamoto A, Tatsuno I, Isaka M, Ohta M: Characterization of a virulence-associated and cell-wall-located DNase of Streptococcus pyogenes. Microbiology 2010, 156:184-190.

25. Aziz RK, Ismail SA, Park HW, Kotb M: Post-proteomic identification of a novel phage-encoded streptodornase, Sda1, in invasive M1T1 Streptococcus pyogenes. Mol Microbiol 2004, 54:184-197.

26. Bozzaro S, Bucci C, Steinert M: Phagocytosis and host-pathogen interactions in Dictyostelium with a look at macrophages. Int Rev Cell Mol Biol 2008, 271:253-300.

27. Dallaire-Dufresne S, Paquet VE, Charette SJ: Dictyostelium discoideum: a model for the study of bacterial virulence. Can J Microbiol 2011, 57:699-707.

28. Lopez-Cortes LF, Cruz-Ruiz M, Gomez-Mateos J, Jimenez-Hernandez D, Palomino J, Jimenez E: Measurement of levels of tumor necrosis factor-alpha and interleukin-1 beta in the CSF of patients with meningitis of different etiologies: utility in the differential diagnosis. Clin Infect Dis 1993, 16:534-539.

29. Leppert D, Leib SL, Grygar C, Miller KM, Schaad UB, Hollander GA: Matrix metalloproteinase (MMP)-8 and MMP-9 in cerebrospinal fluid during bacterial meningitis: association with blood-brain barrier damage and neurological sequelae. Clin Infect Dis 2000, 31:80-84

30. Azeh I, Mader M, Smirnov A, Beuche W, Nau R, Weber F: Experimental pneumococcal meningitis in rabbits: the increase of matrix metalloproteinase-9 in cerebrospinal fluid correlates with leucocyte invasion. Neurosci Lett 1998, 256:127-130.

31. Chang A, Khemlani A, Kang H, Proft T: Functional analysis of Streptococcus pyogenes nuclease $A(S p n A)$, a novel group $A$ streptococcal virulence factor. Mol Microbiol 2011, 79:1629-1642.

32. Sumby P, Barbian KD, Gardner DJ, Whitney AR, Welty DM, Long RD, Bailey JR, Parnell MJ, Hoe NP, Adams GG, DeLeo FR, Musser JM: Extracellular deoxyribonuclease made by group A Streptococcus assists pathogenesis by enhancing evasion of the innate immune response. Proc Natl Acad SCi US A 2005, 102:1679-1684.

33. Brinkmann V, Reichard U, Goosmann C, Fauler B, Uhlemann Y, Weiss DS, Weinrauch Y, Zychlinsky A: Neutrophil extracellular traps kill bacteria. Science 2004, 303:1532-1535.

doi:10.1186/1756-0500-7-424

Cite this article as: Haas et al:: Characterization of DNase activity and gene in Streptococcus suis and evidence for a role as virulence factor. BMC Research Notes 2014 7:424 